\title{
Development and Application of Transmission Line Conductor and Grounding Foreign Body Removal Device
}

\author{
Zhao Hui, Wang Wei, Zhu Yuanda, Zhang Su, Shi Xingcai, Jin Yi, Xu Minghu, \\ Liu Jun, Yi Yongfei, Jin Xin
}

The Technical Training Center, Liaoning Electric Power Company Limited, State Grid, China.

fushunpowersupply@163.com

Keyword: Transmission Line, live working, foreign body, removal device, develop

\begin{abstract}
This paper describes the development of a new transmission line live working foreign body removal, a tool developed by a lightweight safe, efficient, timely treatment and threats to the safe operation of overhead transmission lines foreign body proved to avoid the strong electric field of charged workers enter the job, with high practical value and promote sense of professional and technical personnel for live operator and maintenance applications.
\end{abstract}

\section{Introduction}

Due to the long-running transmission lines under natural conditions, so it will inevitably be affected by the external environment, such as in the coastal areas of particular climate impact of the typhoon on the foreign body toppled transmission line conductor; towns and villages along the line after the annual Spring Festival and Lantern Festival, residents of villages along the line fireworks, the remnants of firecrackers on the line parachute hanging wire, these phenomena directly affect the safe operation of the transmission lines, if it not in time will produce a fault line trip, in the past to deal with these defects are relying on artificial lines strong electric field of charged wire processing, high labor intensity of workers, high security risk, so in this case it must innovate to develop a so workers do not enter the lightweight high pressure electric field can play a quick and easy tool for the treatment effect. More timely and effective zone electric transmission lines to solve $35-220 \mathrm{kV}$ guide, the online presence of foreign material conditions endanger the safe operation of the power grid, power transmission lines to ensure the reliability of the power grid for much less damage play a positive role[1-3].

\section{Metallic material selected}

When taking into account the weight of the entire foreign body removal, in terms of design choice using lightweight aluminum alloy sheet as the main structural cleaner base in considering rotating disk rotating gears, opt for the more wear-resistant metal steel precision gears, in order to make the whole Turn the base plate can be flexible, lightweight spin rotation, considered in the design of the base rotating disk to install the bearing rolling on the ground insulation can make the base rotating disk transfer operation when the rope cutting blade rotating flexible drive turntable were cut for foreign body removal purposes. The entire foreign body removal blade designed for cutting the main features crescent-type serrated blade, taking into account the foreign body can not be maintained at the site immobile state and foreign feature different circumstances.

\section{Design of the overall structure}

In recent years, foreign body characterized by transmission lines hanging survey analysis, foreign body hanging in the online guide to not maintain eternal stationary condition (due on cables erected in the wild space) and the characteristics of foreign objects of varying lengths, so rotation on the ground led rope insulation under: Consider cleaner design is divided into three vertical wheel structure, three wheel rotation function, the first wheel features: hanging scroll in 
leading the line, the second wheel function the second round of the guide wheel drive ground vertical crescent-style jagged blade through the central axis of the wheel, to pull off the foreign body cutting function. The third wheel functions: on the ground led rope insulation under the central axis of rotation of the wheel driven by the third round with a guide wheel base ground level cutting blade rotating disk drive turntable rotation were cut for foreign body removal purposes[4,5].

Turn the control disc bottom rope, designed for long oval hole to the edge of the rotating disc, a diameter of about $\varnothing 8-\varnothing 10$ insulation rope to pass through is reasonable.

Entire foreign body removal device designed to split into two functional use, suspension assembly according to the site characteristics selected foreign body (the second round) vertical features cutting and that (third round) level features cutting.

In order to facilitate the potential electrical insulating operating rod operation, cleaner design on cables hanging body of a movable wheel side door panel in place to consider adding a vertical position welding small circle diameter of about $\varnothing 16 \mathrm{~mm}$ to earth potential insulated lever operation after suspension on the guide to online job opened the side door panel removed.

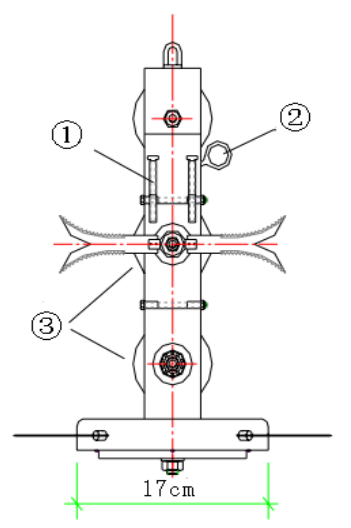

Figure 1 Main view of the body

In the Figure 1, (1) two bolt for fixing the main body, to prevent the side door after the side tilt unstable operating side door is not well off place; (2) side door pull ring; (3) consider whether the two should be replaced with insulated outer aluminum pulley sheaves.

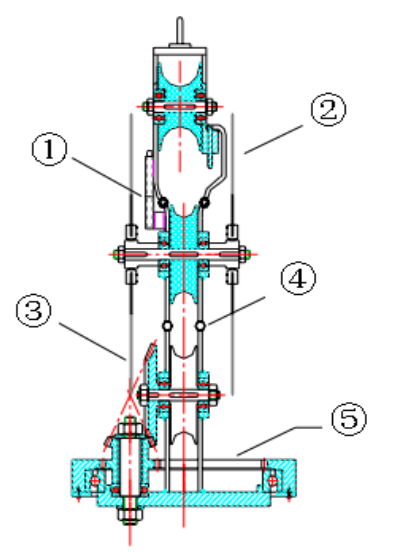

Figure 2 Left sectional of the body

In the Figure 2, (1) using small bolts after disassembling the plug can be connected to the lower end of the upper end of the function. (2) two blades must be kept in place after the body balance and tighten. (3) cutting blade and do not touch the lower gear to maintain sufficient clearance so that the blade can turn normal. (4) Upper and lower sections detach ably node. (5) consider replacing the entire chassis unit for aluminum rotating disc, reduce overall weight, easy to pick potential insulating operating rod hanging on the wire. 


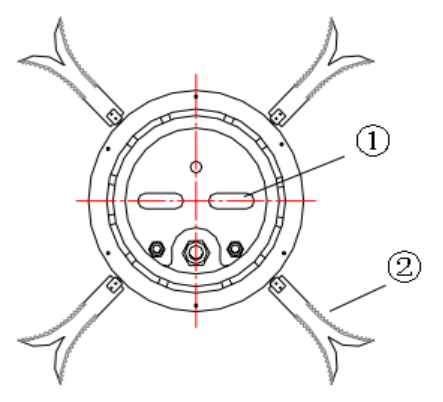

Figure 3 Bottom view of the body

In the Figure 3, (1) changed to the original single-hole rectangular oval hole. (2) steel blade must have a certain steel degrees.

\section{The portability of tool}

1) Replacement tool package weight of all the major components were $4.6 \mathrm{~kg}$; package components lighter; split on part weight $1.35 \mathrm{~kg}$; split lower part weight $3.25 \mathrm{~kg}$, transportation site easy to use.

2) The entire foreign body removal tool, install only the operator two people; complete a cleanup operation takes about 20min; compared with the previously used other potential job processing time and work efficiency significantly improve effectiveness.

\section{The effect of the practical application}

By sending electric field of charged duty officers repeatedly tried and practical operation, that the use of the foreign body removal tool has the following advantages:

1) This kit high mechanical strength, safety factor, can promptly and effectively resolve $35-220 \mathrm{kV}$ zone electric transmission line guide, the online presence of foreign material conditions endanger the safe operation of the power grid, power transmission line to ensure the reliability of the grid and more for less damage play a positive role in enhancing the processing guide to online foreign body hanging in the efficiency, safety and reliability.

2) Convenient to carry the tools and instruments, replace insulation and insulation ladder shielding clothing and other industrial equipment traditionally used, with easy installation; simple operation and reduce the potential of high-altitude operations, such as labor intensity, field operations process safe and easy and so on.

Figure 4 and Figure 5 are the previous and present methods separately.

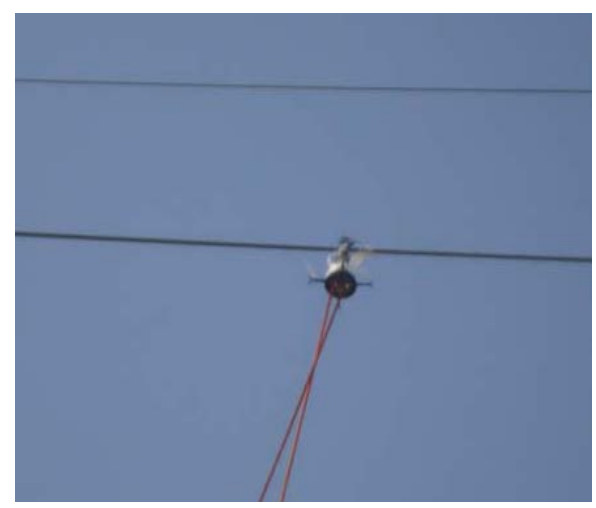

Figure 4 Present approach 


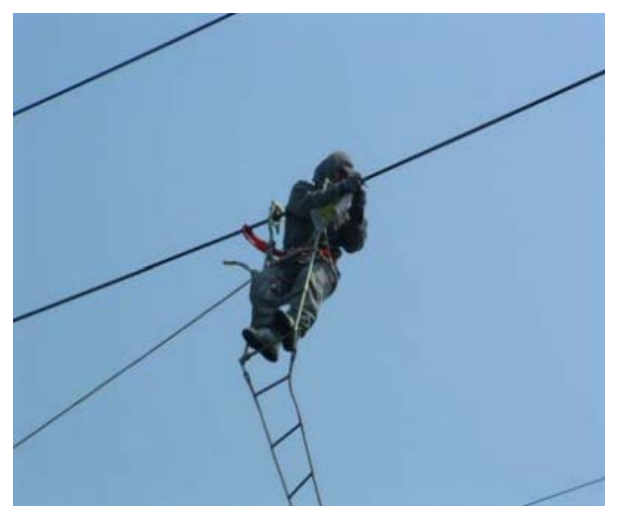

Figure 5 Primary approach

\section{Conclusion}

Development and application of the tools and instruments to solve the existence $35-220 \mathrm{kV}$ transmission overhead lines and the ground presence of foreign bodies in the past to take other potential job law to bring the labor intensity of workers and job security risks and other issues, through the development of tools and instruments to take to improve potential practices to enhance job security and reduce labor intensity and the workers live fast processing defects substantive issues, the biggest problem is to solve the workers to avoid entering a strong electric field, to avoid operational risk, hanging in the guide to improve the processing efficiency of online foreign body and safety and reliability. The actual use of the effect is obvious, and fully achieves Research practical work to solve production problems purposes, effectively improve the efficiency and safety objectives to ensure the safety and reliability of transmission power lines.

\section{References}

[1] Wang Qingkui. Transmission line operation and maintenance. China Electric Power Press, 2003.

[2] State Grid Corporation of electricity safety regulations (power line section). China Electric Power Press, 2003.

[3] Ying Wei. Overhead transmission line condition-based maintenance and practical technology. China Electric Power Press, 2004

[4] Three live working procedures. China Electric Power Press, 2003.

[5] Chen Tianxiang, Wang Yinzhong. Electrical Test. Beijing: China power press, 2010 